\title{
The relationship between collapsibility index of inferior vena cava and hypotension after spinal anesthesia
}

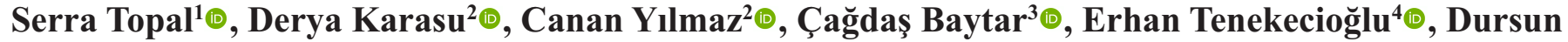 \\ Topal $^{5} \odot$
}

${ }^{1}$ Department of Anesthesiology and Reanimation, Bursa City Hospital, Bursa, Turkey

${ }^{2}$ Department of Anesthesiology and Reanimation, University of Health Sciences, Bursa Yuksek Ihtisas Training and Research Hospital, Bursa, Turkey

${ }^{3}$ Department of Anesthesiology and Reanimation, Gemlik State Hospital, Bursa, Turkey

${ }^{4}$ Department of Cardiology, University of Health Sciences, Bursa Yuksek Ihtisas Training and Research Hospital, Bursa, Turkey

${ }^{5}$ Department of Cardiology, Bursa City Hospital, Bursa, Turkey

\section{ABSTRACT}

Objectives: Hypotension is a common complication of spinal anesthesia. Imaging of inferior vena cava (IVC) and measurement of the IVC-collapsibility index (IVC-CI) by ultrasonography (USG) has been a widely used non-invasive, easy and reliable method for measurement of the fluid imbalance. In the present study, we aimed to investigate the predictive ability of the maximum IVC diameter (dIVCmax) and IVC-CI for hypotension after spinal anesthesia.

Methods: The study was designed as prospective and observational. One hundred thirty-two patients aged 1875 years with ASA I-II underwent inguinal hernia surgery with spinal anesthesia and recruited to the study. Maximum and minimum (dIVCmin) IVC diameters were measured. IVC-CI (\%) was quantified according to the formula of $[(\mathrm{dIVCmax}-\mathrm{dIVCmin}) / \mathrm{dIVKmax}] \times 100 \%$.

Results: The patients were grouped as hypotensive and non-hypotensive. In fifty-seven patients of 120 cases $(47.5 \%)$, hypotension has emerged following spinal anesthesia. No significant differences in dIVCmax and IVC-CI were recorded between the study groups $(p>0.05)$. There were significant inverse correlation between age and IVC-CI. Significant positive correlation between the lowest values of the systolic arterial pressure, diastolic arterial pressure, mean arterial pressure and IVC-CI and significant positive correlation between dIVCmax and diastolic blood pressure, maximum and minimum values of the mean arterial pressure.

Conclusions: We found that dIVCmax and IVC-CI values measured before spinal anesthesia were not sufficient parameters enough to predict hypotension after spinal anesthesia. Further studies investigating the IVC measurements under spinal anesthesia together with dynamic hemodynamic monitorization modalities are needed.

Keywords: Hypotension, inferior vena cava, inferior vena cava collapsibility index, spinal anesthesia, ultrasonography

$\mathrm{H}$ ypotension is the most common complication of spinal anesthesia. In the literature, hypotension

is reported at a ratio of $8.2-57.9 \%$. The primary underlying cause of hypotension induced by spinal anesthe-

Received: May 21, 2020; Accepted: November 13, 2020; Published Online: January 21, 2021

How to cite this article: Topal S, Karasu D, Yılmaz C, Baytar Ç, Tenekecioğlu E, Topal D. The relationship between collapsibility index of inferior vena cava and hypotension after spinal anesthesia. Eur Res J 2021;7(3):319-325. DOI: 10.18621/eurj.739559

Address for correspondence: Serra Topal, MD., Bursa City Hospital, Department of Anesthesiology and Reanimation, Doğanköy/Nilüfer, Bursa, Turkey E-mail:dr.serra@msn.com,Tel:+902249750000,Fax:+902242680062 
sia (SA) is arterial, arteriolar and venous dilatation due to preganglionic sympathetic nerve blockage. The hypotension may aggravate in case of volume depletion in patients underwent spinal anesthesia [1-4].

Measurement of the inferior vena cava (IVC) diameter by ultrasound and IVC-collapsibility index (IVC-CI) by ultrasonography (USG) has been a widely used non-invasive, straightforward and reliable method for assessment of intravascular volume. IVC is a highly compliant vessel and the change in the collapsibility of the vessel can guide the clinicians about the clinical status of the patient. At each respiratory cycle the vessel contacts and expands. With negative pressure at each inspiration, the venous return to the heart increases and IVC collapse. The changes in the diameter of the vessel depends on the total body fluid status. As compared to the relatively higher intravascular volume status, lower volume within the vasculature increases the percentage of the IVC collapse. It has been demonstrated that IVC diameter can change even in healthy subjects however, the maximum diameter of IVC may decrease deeply in hypovolemic cases [5]. The collapsibility of IVC is efficient method for demonstrating the intravascular volume. IVC collapsibility can be calculated using he formula as follows: [(dIVCmax - dIVCmin) / dIVCmax] $\times 100 \%$.

The aim of the current analysis is to investigate the relationship between IVC diameter, hypotension sourced from SA within the cases underwent an inguinal hernia operation and IVC -CI.

\section{METHODS}

The ethics committee of the local institution approved the protocol, and each patient gave written informed consent before inclusion. The study was a prospective observational implemented between 01.04.2017-01.10.2017. The subjects between 18-75 years old with American Society of Anesthesiologists (ASA) Score I, II those were planned to have inguinal hernia operation under spinal anesthesia recruited to the current study. The patient exclusion criteria were as follows; body mass index $>35$, presence of hypertension, major peripheral artery disease, multiple vessel disease, unstable angina, left ventricle ejection fraction $<40 \%$, respiratory disease, neurological disorder, increased intraabdominal pressure, cardiac pacemaker, using angiotensin receptor blocker. The cases with systolic arterial pressure $>150 \mathrm{mmHg}$ and mean arterial pressure $<60 \mathrm{mmHg}$ were excluded from the study.

The demographical data of the patients enrolled into the study were recorded. By a blinded physician,

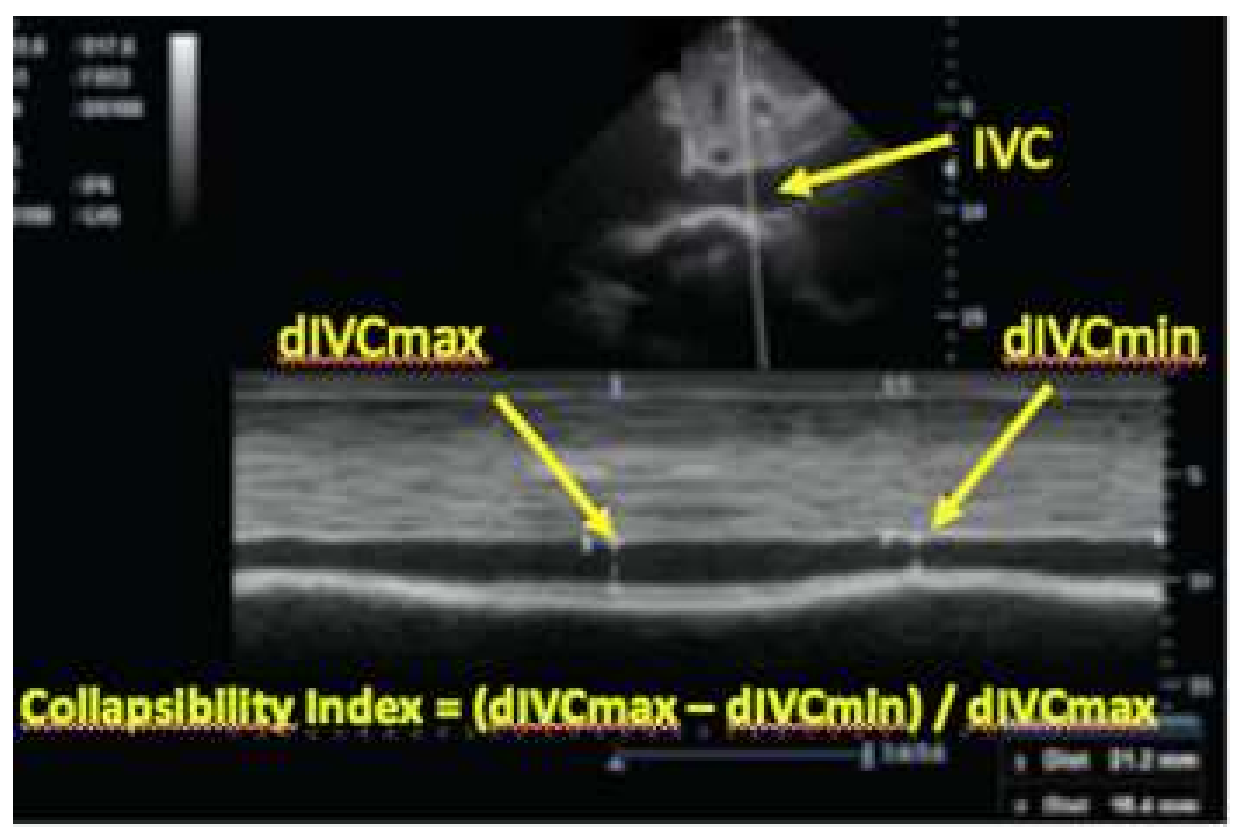

Fig. 1. Ultrasound measurements of inferior vena cava (IVC) and calculation of collapsibility index. Panel above shows twodimensional scan of the IVC with right atrium to the left and panel below shows M-mode scan with respiratory variations in diameter. dIVCmax = maximum diameter of IVC, dIVCmin = minimum diameter of IVC. 
five min prior to spinal anesthesia, the diameters of the IVC at inspiration (dIVCmin) and expiration (dIVCmax) were recorded at 2-3 cm subcostal location using 5-Mhz probe ultrasound (Esaote, MyLab 30gold cardiovascular, Florence, Italy) at M-mode with the patient in the supine position. This process were repeated 3 times and the mean of the 3 measurements were recorded. IVC collapsibility index (IVC-CI) were calculated using the formula of [ dIVCmax - dIVCmin)/dIVCmax $\times \% 100$ ] (Fig. 1). The cases with $>0.2 \mathrm{~cm}$ difference between the two sequential dIVCmax were excluded from the study.

Following the routine monitorization, premedication with intravenous (IV) midazolam 0.03-(0.05 $\mathrm{mg} / \mathrm{kg}$ ) (Dormicum ${ }^{\circledR}$, Deva, Istanbul, Turkey) were implemented by an anesthetist outside the study. At the sitting position, sterilization was done over the skin zone of the spinal anesthesia and around the L4-5 or L3-4 intervertebral spaces with a 25G (gauge) Quincke spinal needle was introduced using mid-line approach. After observing the cerebrospinal fluid flow, 0.5\% hyperbaric bupivacaine (Marcaine Heavy, AstraZeneca, Kurklareli, Turkey) was injected at a dosage according to the height of the patient (12-18 mg). The level of the sensorial block was determined using Pinprick test and the level of the motor block level was defined utilizing Bromage scale. As the block level was reached to $\mathrm{T} 8$ and Bromage 0, the operation started. Following spinal anesthesia, the subjects at whom vasoactive medication was implemented due to hypotension and the dosage of those vasoactive drugs were recorded. After the anesthesia till the surgical operation concluded, the heart beats per min and the blood pressures of the patients were recorded at every $2 \mathrm{~min}$. Also, the maximal sensory levels of the cases were recorded.

No any patients received any fluid replacement therapy before the spinal anesthesia. The fluid therapy was planned calculating the preoperative starvation time and fluid maintenance and target fluid replacement was done during the surgical operation. Fluid replacement regime was implemented as follows; 4 $\mathrm{mL} / \mathrm{h}$ for the first $10 \mathrm{~kg}$ body weight, $2 \mathrm{ml} / \mathrm{h}$ for the second $10 \mathrm{~kg}$ body weight and $1 \mathrm{ml} / \mathrm{h}$ for the rest body weight. Hypotension was defined as the starting mean arterial pressure decreases by $>30 \%$ and/or mean arterial pressure was $<60 \mathrm{mmHg}$. The patients were grouped according to the hypotension status. As hypotension was determined IV fluid infusion was increased. Ephedrine (Ephedrine hydrochloride, Osel, Beykoz, Istanbul) was utilized at a dosage of 5-10 mg. When heart beat per min was $<40$, IV atropine (Atropine Sulfate, Biofarma, Sancaktepe, Istanbul) (0.5 $\mathrm{mg}$ ) was administered.

\section{Statistical Analysis}

For calculating the sample size $G$ power version 3.1.9.2 was used. The effect size for the independent study groups for the t-test was $d=0.57$ and for $\alpha=$ 0.05 with power $=0.85$, the minimum needed case number was 118. For statistical analysis SPSS 22.00 (Statistical Package for Social Sciences, Inc., Chicago,

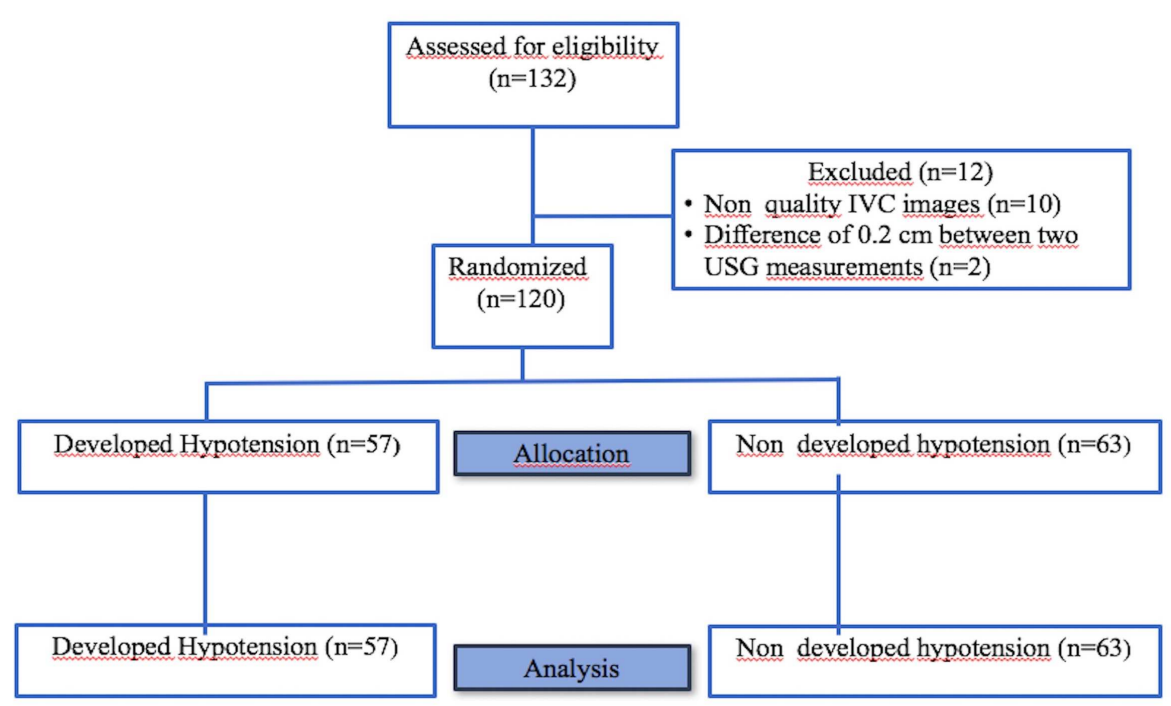

Fig. 2. Flow chart of the study. 
IL) was used. Numerical data are expressed as mean \pm standard deviation or median and inter-quartile range depending on their distribution which was tested by Kolmogorov-Smirnov test. Spearman correlation test was used for the correlation analysis. Continuous variables with normal and non-normal distributions were compared using Student's t-tests and MannWhitney U tests, respectively. Categorical variables were compared using the Pearson's chi-square test or Fischer's exact test, as appropriate.

\section{RESULTS}

A total of 132 patients who underwent inguinal hernia with spinal anesthesia were included in the study and statistical analysis was performed in 120 cases (Fig. 2).

The patient characteristics are shown in Table 1. Hypotension was recorded in $47.5 \%(\mathrm{n}=57)$ of the patients. No significant difference was noted for dIVCmax and IVC-CI between the study groups (Table 1). Sixty five percent of the cases have rendered dIVC $<15 \mathrm{~cm}$ and IVC-CI $>50 \%$ whereas in $2.5 \%$ of the subjects, IVC diameter was $15-25 \mathrm{~mm}$ and IVC-CI > $50 \%$.

There was no meaningful correlation between dIVCmax and IVC-CI $(\mathrm{r}=0.145, p=0.114)$. No statistically significant negative correlation was recorded between age and IVC-CI ( $p<0.05, \mathrm{r}=0.184)$ (Table 2 ). No significant correlation was noted between the percentage of the decrease in mean arterial pressure and IVC-CI and dIVCmax (Fig. 3).

\section{DISCUSSION}

The researchers investigated the impact of USG guided IVC measurement on the hypotension after spinal anesthesia in patients undergoing inguinal hernia operation with a prospective observational study. In $47.5 \%$ of the cases hypotension during the spinal anesthesia has been recorded. There was no statistically significant difference between the cases in terms of hypotension after spina anesthesia related with dIVCmax and IVC-CI. While there was a negative correlation between age and IVC-CI, no significant correlation was observed between the percentage of

Table 1. Comparison of patient characteristics, hemodynamic data, and preoperative inferior vena cava ultrasound measurements between patients who did and did not develop hypotension after spinal anesthesia

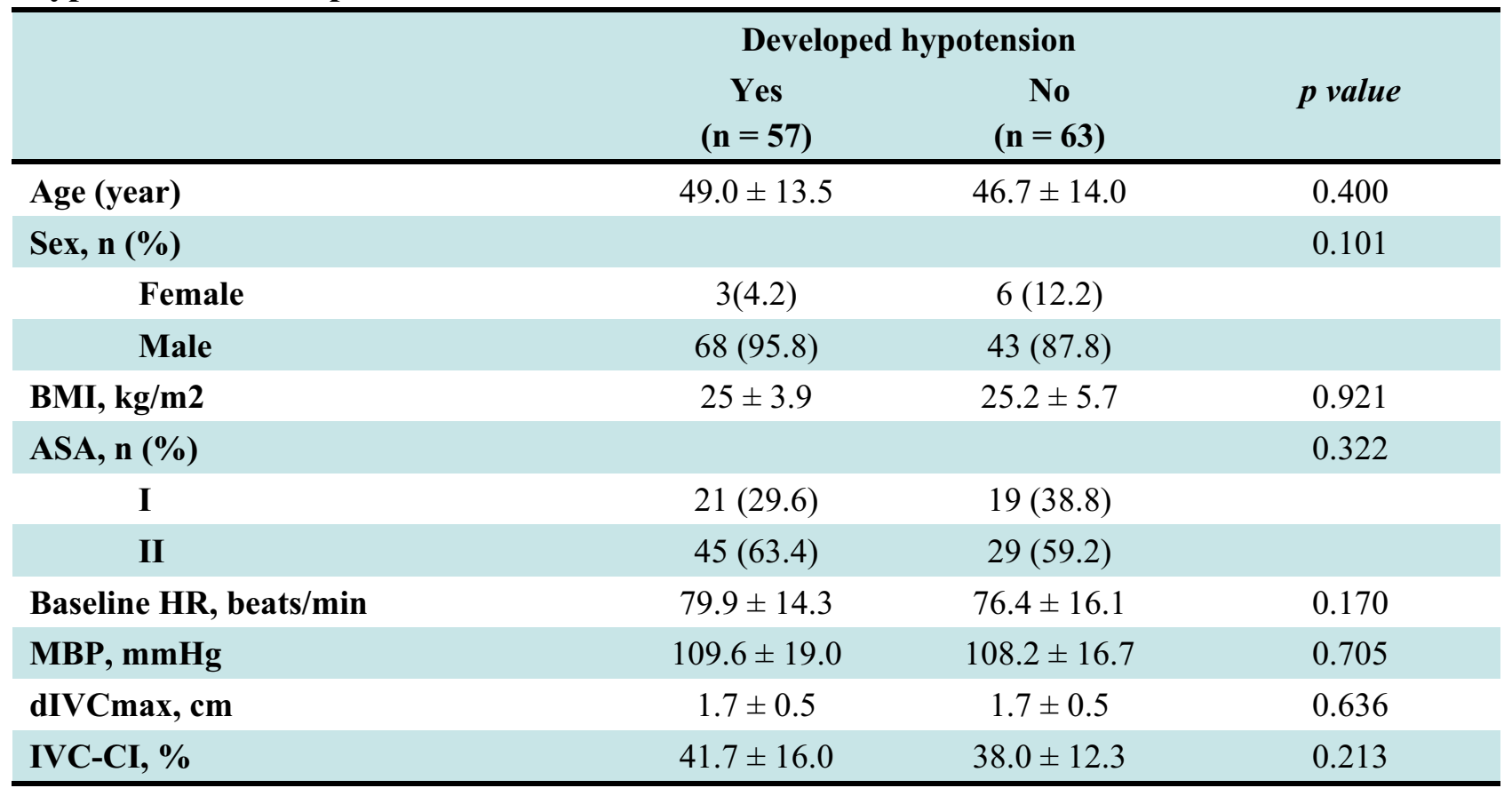

$\mathrm{ASA}=$ American Society of Anesthesiologists, BMI = Body Mass Index, MBP $=$ Mean Blood Pressure, $\mathrm{dIVCmax}=$ Inferior Vena Cava Maximum Diameter, IVC-CI = Inferior Vena Cava Collapsibility Index 


\section{Table 2. Comparison of patient characteristics between inferior vena cava maximum diameter and inferior vena cava collapsibility index}

\begin{tabular}{lcccc}
\hline & \multicolumn{2}{c}{$\begin{array}{c}\text { dIVCmax } \\
\text { (cm) }\end{array}$} & \multicolumn{2}{c}{$\begin{array}{c}\text { IVC-CI } \\
\text { (\%) }\end{array}$} \\
\hline Age (year) & \multicolumn{1}{c}{ r } & $p$ value & $\mathrm{r}$ & $p$ value \\
Weight (kg) & 0.114 & 0.215 & -0.184 & 0.044 \\
Height (cm) & -0.158 & 0.085 & 0.173 & 0.059 \\
BMI (kg/m2) & -0.124 & 0.177 & 0.123 & 0.181 \\
\hline
\end{tabular}

$\mathrm{BMI}=$ Body Mass Index, $\mathrm{dIVCmax}=$ Inferior Vena Cava Maximum Diameter, IVC-CI $=$ Inferior Vena Cava Collapsibility Index
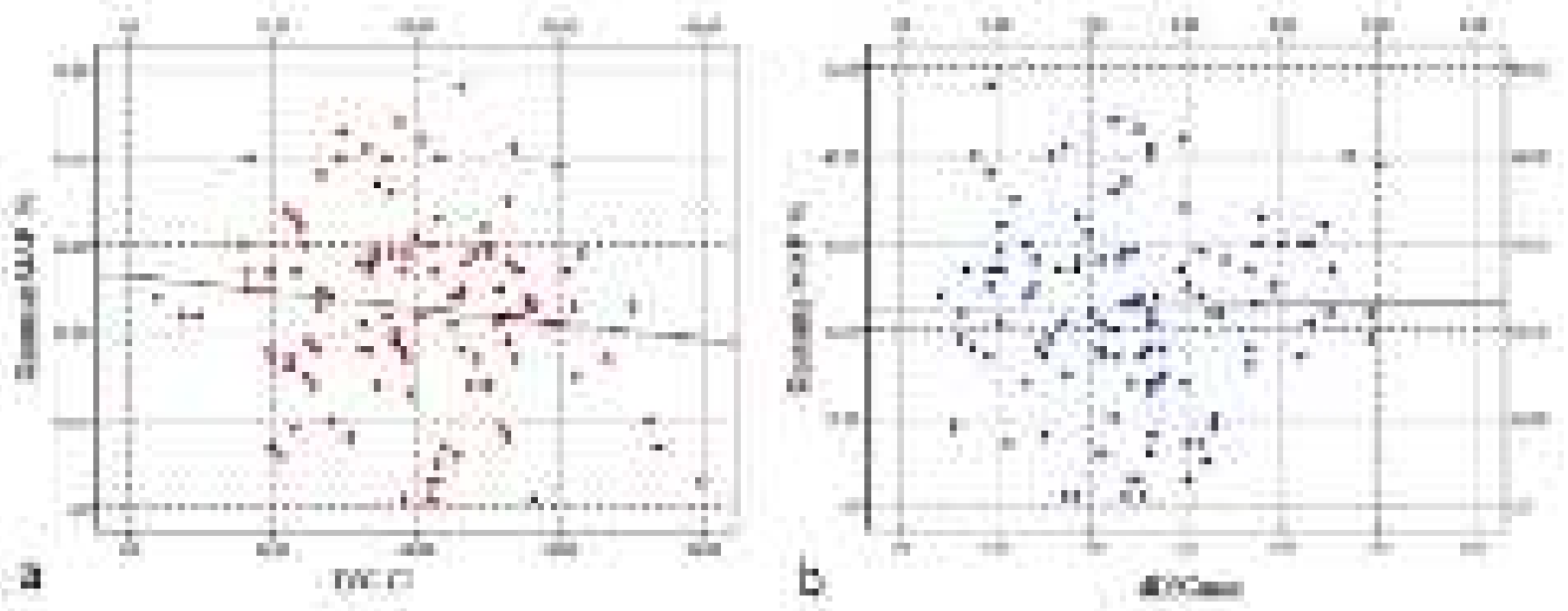

Fig. 3. (a) Correlation between the percentage of the decrease in mean arterial pressure (MAP) and inferior vena cava collapsibility index (IVC-CI), (b) Correlation between the percentage of the decrease in mean arterial pressure (MAP) and inferior vena cava maximum diameter (dIVCmax).

fall in MAP and IVC-CI and dIVCmax.

IVC ultrasound has been a frequent, easily doable and non-expensive method for assessment of intravascular volume status. Regarding the importance of determination of the pre-operative volume status, fast evaluation by ultrasonography may provide benefit in the treatment of critical patients [5]. While inspiration induces IVC collapse by enabling a negative pressure within the thorax, expiration allows an increment in venous return that yields the return of the IVC diameter to its original size. The decrease in the intravascular volume status may increase the percentage of the IVC collapse. In the children with dehydration needing volume supplement, the IVC diameter was decreased [6,
7]. Relatively higher IVC-CI, in particular with a small IVC diameter shows a decreased volume status [8] .According to the Society of Emergency Physicians, in adult patients with normal CVP, IVC dimeter and IVC-CI should be $15-25 \mathrm{~mm}$ and $>50 \%$ whereas in cases with volume deficient due to total compression the diameter of the IVC was less than $15 \mathrm{~mm}$ [9]. In our study, in $65 \%$ of the cases IVC diameter was $<15$ $\mathrm{mm}$ and IVC-CI was $>50 \%$ while in $2.5 \%$ of the subjects the diameter of the IVC was 15-25 mm and IVCCI was $>50 \%$. By those results, it was assumed that there was volume deficit in most of our patients. However, since there was no monitorization of the CVP, no relation could be detected between the CVP and the 
volume status.

Ceruti et al. [10] has reported the results of 160 cases about the IVC diameter and IVC collapsibility by ultrasound preceding spinal anesthesia. In one group within 15 min $500 \mathrm{ml}$ fluid (Ringerfundine; B. Braun) was loaded and IVC-CI was evaluated and following 15 min volume replacement spinal anesthesia was implemented. Finally, before the spinal anesthesia, volume loading by the guidance of IVC USG decreased the incidence of arterial hypotension, provided the volume status optimization and decreased the interventions following arterial hypotension attacks. However, no any correlation has been demonstrated between the volume loading preceding the spinal anesthesia and IVC-CI [10]. Beside that study, no ant subject received pre-operative volume replacement. Calculating the pre-operative starvation time and hourly volume replacement requirement, crystalloid replacement was applied. There was no any significant relationship between the amount of volume loaded and hypotension, in the present study. In line with the study by Ceruti et al. [10], no any meaningful correlation was detected between the IVC-CI and the hypotension after spinal anesthesia, in the current study.

Zhang et al. [11] has demonstrated that for the hypotension after general anesthesia, IVC measurements before induction of general anesthesia, IVC-CI was more predictive than dIVCmax and in the same study the threshold value was $43 \%$ for IVC-CI. In the present study, there was no significant difference in IVCCI between the study groups and IVC-CI was not found predictive for hypotension after spinal anesthesia. The underlying cause for such result might be multifactorial component of the hypotension after spinal anesthesia in which hypovolemia could be one of them. While IVC-CI is so determinant for the stats of intravascular volume, it doesn't seem the same as for hypotension after spinal anesthesia.

Intra-operative hypotension is one of the frequent adverse event for anesthesia, whereas the definition may change within the clinical trials. Bjiker et al. [12] [has found 140 definitions for hypotension in the literature. In our study, similar to the study of Zhang et al. [11] , hypotension was defined as $>30 \%$ decrease in mean arterial pressure and/or a value of $<60 \mathrm{mmHg}$ in mean arterial pressure. The sympathetic blockage following spinal anesthesia impacts of the regulation of the circulation both by decreasing the venous return and also decreasing the systemic vascular resistance. By the way, as the level of blockage reaches to T4, the cardioselective fibers also are impeded, heart rhythm and also the cardiac output may reduce [13]. Since the sympathetic fibers are within T1-L2, the blockages under the L2 don't have influence on arterial blood pressure [14] . In the present study, as the block level was T8, the surgical operation was initiated. The level of the blockage didn't reach to T4 in any patient within our study. There was no significant difference in the highest level of the blockage between the cases with and without hypotension after spinal anesthesia in the present study.

\section{Limitations}

The limitations of our study are as follows; onecenter design of the study, the nature of the data came from only one type of surgical operation, no measurement of IVC during the hypotension and after the spinal anesthesia, no comparison of the IVC measurements with some dynamic tests such as CVP and cardiac output. Another limitation was several definitions of the hypotension. The cases with the history of hypertension were not included but the subjects with undiagnosed hypertensive subjects might be recruited.

\section{CONCLUSION}

IVC-CI and dIVCmax were not significantly associated with the hypotension after spinal anesthesia in the patients undergoing inguinal hernia operation with spinal anesthesia. Further studies investigating the IVC measurements under spinal anesthesia together with dynamic hemodynamic monitorization modalities are needed.

\section{Authors' Contribution}

Study Conception: ST, DK; Study Design: SBT, CY; Supervision: ST, ÇB; Funding: ÇB, DT; Materials: DT, CY; Data Collection and/or Processing: DK, CY; Statistical Analysis and/or Data Interpretation: ET, DK; Literature Review: ÇB, DT; Manuscript Preparation: ST, ET and Critical Review: ST, DT.

\section{Conflict of interest}

The authors disclosed no conflict of interest during the preparation or publication of this manuscript. 


\section{Financing}

The authors disclosed that they did not receive any grant during conduction or writing of this study.

\section{REFERENCES}

1. Lee JE, George RB, Habib AS. Spinal-induced hypotension: Incidence, mechanisms, prophylaxis, and management: summarizing 20 years of research. Best Pract Res Clin Anaesthesiol 2017;31:57-68.

2. Baraka A, Taha S, Ghabach M, Sibaii A, Nader A, Matta M. Hypertonic saline prehydration in patients undergoing transurethral resection of the prostate under spinal anaesthesia. Br J Anaesth 1994;72:227-8.

3. Shimosato S, Etsten BE. The role of the venous system in cardiocirculatory dynamics during spinal and epidural anesthesia in man. Anesthesiology 1969;30:619-28.

4. Critchley LAH, Short TG, Gin T. Hypotension during subarachnoid anaesthesia: haemodynamic analysis of three treatments. Br J Anaesth 1994;72:151-5.

5. Dipti A, Soucy Z, Surana A, Chandra S. Role of inferior vena cava diameter in assessment of volume status: a meta-analysis. Am J Emerg Med 2012;30:1414-9.e1.

6. Brennan JM, Blair JE, Goonewardena S, Ronan A, Shah D, Vasaiwala $\mathrm{S}$, et al. Reappraisal of the use of inferior vena cava for estimating right atrial pressure. J Am Soc Echocardiogr 2007;20:857-61.
7. Brennan JM, Ronan A, Goonewardena S, Blar JEA, Hammes $\mathrm{M}$, Shah D, et al. Handcarried ultrasound measurement of the inferior vena cava for assessment of intravascular volume status in the outpatient hemodialysis clinic. Clin J Am Soc Nephrol 2006; 1:749-53.

8. Seif D, Mailhot T, Perera P, Mandavia D. Caval sonography in shock: a noninvasive method for evaluating intravascular volume in critically ill patients. J Ultrasound Med 2012;31:1885-90. 9. Wong SP . In Otto CM (ed.), Textbook Clinical Echocardiography, 2nd. Ed., Philadelphia: WB Saunders, 2000

10. Ceruti S, Anselmi L, Minotti D, Franceschini D, Aguirre J, Borgoet A, et al. Prevention of arterial hypotension after spinal anaesthesia using vena cava ultrasound to guide fluid management. Br J Anaesth 2018;120:101-8.

11. Zhang J, Critchley LAH. Inferior vena cava ultrasonography before general anesthesia can predict hypotension after induction. Anesthesiology 2016;124:580-9.

12. Bijker JB, van Klei WA, Kappen TH, van Wolfswinkel L, Moons KGM, Kalkman CJ. Incidence of intraoperative hypotension as a function of the chosen definition. Anesthesiology 2007; 107:213-20.

13. Hartmann B, Junger A, Klasen J, Benson M, Jost A, Banzhaf $A$, et al. The incidence and risk factors for hypotension after spinal anaesthesia induction: an analysis with automated data collection. Anesth Analg 2002;94:1521-9.

14. McCrae AF, Wildsmith JA. Prevention and treatment of hypotension during central neural block. Br J Anaesth 1993;70:67280 . 